\title{
RETRACTED ARTICLE: Human MTERF3 Crystal Structure Forms a Left-Handed Superhelix
}

Dong-Uk Kim, Sang-Gil Cho, Kug-Lae Kim, and Hyun-Soo Cho*

This article has been retracted due to unethical behavior by the authors. 